\title{
Localization for a Class of One Dimensional Quasi-Periodic Schrödinger Operators
}

\author{
J. Fröhlich ${ }^{1}$, T. Spencer ${ }^{2}$, and P. Wittwer ${ }^{3, \star}$ \\ 1 Theoretical Physics, E.T.H., Zürich, Switzerland \\ ${ }^{2}$ School of Mathematics, I.A.S., Princeton, NJ 08540, USA \\ ${ }^{3}$ Department of Mathematics, Rutgers University, New Brunswick, NJ 08903, USA
}

Dedicated to Res Jost and Arthur Wightman with respect and affection

Abstract. We prove for small $\varepsilon$ and $\alpha$ satisfying a certain Diophantine condition the operator

$$
H=-\varepsilon^{2} \Delta+\frac{1}{2 \pi} \cos 2 \pi(j \alpha+\theta) \quad j \in \mathbb{Z}
$$

has pure point spectrum for almost all $\theta$. A similar result is established at low energy for $H=-\frac{d^{2}}{d x^{2}}-K^{2}(\cos 2 \pi x+\cos 2 \pi(\alpha x+\theta))$ provided $K$ is sufficiently large.

\section{Introduction}

In this paper we shall study some of the spectral properties of the operator

$$
H_{c}(\theta)=-\frac{d^{2}}{d x^{2}}+K^{2} v(x, \theta)
$$

acting on $L_{2}(R)$, where

$$
v(x, \theta)=-\cos 2 \pi x-\cos 2 \pi(\alpha x+\theta) .
$$

We shall also study its finite difference approximation on $l_{2}(\mathbb{Z})$ given by

$$
H(\theta)=-\varepsilon^{2} \Delta+v(j, \theta)=-\varepsilon^{2} \Delta+\frac{1}{2 \pi} \cos 2 \pi(\alpha j+\theta) .
$$

In one dimension the finite difference Laplacian has matrix elements $\Delta_{i j}=1$ if $|i-j|=1$ and $\Delta_{i j}=0$ otherwise. When $\alpha$ is rational the spectra of $H$ and $H_{c}$

$\bar{\star}$ Present address: Université de Genève, Switzerland 
are known to be purely absolutely continuous and their generalized eigenstates are Bloch waves $\psi(x)=e^{i k x} p(x)$, where $p$ is a periodic function of $x$. We shall consider the case where $\alpha$ is irrational and $K$ is large or $\varepsilon$ is small. In addition, $\alpha$ is assumed to satisfy the following Diophantine condition:

$$
|n \alpha|_{1} \geq C_{1}^{2} / n^{2} \quad n \neq 0 .
$$

Here $|\theta|_{1}$ is the absolute value of $\theta$ modulo 1 defined so that $0 \leq|\theta|_{1} \leq \frac{1}{2}$. Weaker conditions on $\alpha$ would suffice, but we shall restrict $\alpha$ as above for convenience. We first discuss the finite difference operator.

Theorem 1.1. Let $\alpha$ be as in (1.4). For $\varepsilon$ sufficiently small and $\theta \in \mathbb{R} / \mathbb{Z}$ belonging to a set of measure one, $H$ has pure point spectrum, with eigenfunctions which decay exponentially fast.

This theorem has also been independently established by Sinai [1]. Our proof also applies if the cosine is replaced by any $C^{2}$ periodic function $f$ which is even and has exactly two critical points which are nondegenerate. Returning to the special case (1.3), if $\alpha$ is irrational, then for $2 \pi \varepsilon^{2}<1 / 2$ it is known that $H$ has no absolutely continuous spectrum $[2,3,4]$ and when $2 \pi \varepsilon^{2}>1 / 2$, $H$ has no point spectrum [2,5]. If $2 \pi \varepsilon^{2}<1 / 2$ and $\alpha$ irrational but very well aproximated by rationals, i.e. if $\alpha$ is Liouville, then the spectrum of $H$ is purely singular continuous $[3,6]$. The existence of some point spectrum for $\varepsilon$ small and $\alpha$ sufficiently irrational has been established in [7].

In the continuum it is a classic theorem of Dinaburg and Sinai [8] that, for any $K, H_{c}$ has many Bloch wave eigenstates $\psi(x)=\mathrm{qp}(x) e^{i k x}$, where $\mathrm{qp}$ is quasi-periodic in $x$. These eigenstates correspond with the presence of absolutely continuous spectrum. Our next theorem shows that if $K$ is sufficiently large $H_{c}$ has pure point spectrum at low energy.

Theorem 1.2. Let $\alpha$ be as in (1.4). If $K$ is sufficiently large, then for almost all $\theta$, the spectrum of $H_{c}$ in the interval $\left[-2 K^{2},-2 K^{2}+10 K \sqrt{1+\alpha^{2}}\right]$ is pure point. The eigenfunctions decay exponentially fast.

This theorem provides the first existence proof for eigenstates of $H_{c}$. From general considerations it is known that the spectra of $H$ and $H_{c}$ are essential. Although many of the techniques and results described above work only for the lattice we shall see that our analysis of $H_{c}$ is nearly identical to that of $H$. When $K$ is small, the operator "dual" to $H_{c}$ has been shown to have pure point spectrum [9] by methods closely related to those described here. Moreover the estimates of [9] together with the ideas in [2] or [5] easily imply that $H_{c}$ has no point spectrum for small $K$. We conjecture that $H_{c}$ has purely absolutely continuous spectrum in this case. For large $\varepsilon, H$ is also believed to have only absolutely continuous spectrum.

Determining the nature of the spectrum of $H$ can be viewed as a small divisor problem. Small divisors appear in our analysis of the Green's function $G\left(E_{*}\right)=\left(H-E_{*}\right)^{-1}$ because there are eigenvalues $E^{i}$ of $H$ which come arbitrarily close to $E_{*}$. To overcome this problem we develop a multiscale perturbation scheme in which we keep track of the "location" of the small divisors $[10,12]$. For a fixed energ $E_{*}$ and scale $n$, the location of the small divisors is given by 
a family of disjoint singular intervals $S_{n}\left(E_{*}, \theta_{*}\right)=\left\{I_{n}^{i}\right\}$ defined below. These are certain intervals of length $l_{n} \geq l_{n-1}^{2}$, such that $H(\theta *)$ restricted to $I_{n}^{i}$ with Dirichlet boundary conditions [denoted $H\left(I_{n}^{i}, \theta_{*}\right)$ ] has an eigenvalue $E_{n}^{i}\left(\theta_{*}\right)$ such that for $n \geq 1$ and some fixed $\beta>0$,

$$
\left|E_{n}^{i}-E_{*}\right| \leq \delta_{n} \equiv \exp \left(-\beta l_{n}^{2 / 3}\right)
$$

The uniqueness of $E_{n}^{i}$ is proved in Sect. 4. See Sect. 2 for the precise definition of $S_{n}$. Many of the ideas appearing here were first used in the case where $v(j)$, $j \in \mathbb{Z}^{d}$, are independent random variables $[10,11]$. Here however, the randomness only appears in the parameter $\theta$, and the $v(j)$ are highly dependent.

If an interval $\Lambda$ does not meet any member of $S_{n}\left(E_{*}, \theta_{*}\right)$ then the Green's function for $H(\Lambda)$

$$
G_{\Lambda}\left(E_{*}, x, y\right)=\left[H(\Lambda)-E_{*}\right]^{-1}(x, y)
$$

decays exponentially fast, provided $|x-y| \geq l_{n}$ and $\left|\theta-\theta_{*}\right|_{1} \leq \delta_{n} / 3$. See Theorem 2.2 for a precise statement, and Appendix A for the proof.

The key observation, which uses the fact that there is only one incommensurate frequency $\alpha$, is that the centers $c_{n}^{i} \in \mathbb{Z}$ of the intervals $I_{n}^{i}$ belonging to $S_{n}\left(E_{*}, \theta_{*}\right)$ satisfy the following relation for each $\theta_{*}$ :

$$
m\left(c_{n}^{i}, c_{n}^{j}\right) \equiv \min \left\{\left|\left(c_{n}^{i}-c_{n}^{j}\right) \alpha\right|_{1},\left|\left(c_{n}^{i}+c_{n}^{j}\right) \alpha+2 \theta_{*}\right|_{1}\right\} \leq 4 \delta_{n}^{1 / 2}
$$

See Theorem 2.1. The reason why $m\left(c_{n}^{i}, c_{n}^{j}\right)$ is small is the following. If $H\left(I_{n}^{i}, \theta_{*}\right)$ and $H\left(I_{n}^{j}, \theta_{*}\right)$ have eigenvalues which nearly coincide, (as in (1.5)) we will show that the potential restricted to $I_{n}^{i}$ or $I_{n}^{j}$ must either be nearly translates of each other, in which case $\left|\left(c_{n}^{i}-c_{n}^{j}\right) \alpha\right|_{1}$, is small or they are nearly reflections of each other, so that $\left|\left(c_{n}^{i}+c_{n}^{j}\right) \alpha+2 \theta_{*}\right|_{1}$ is small. This will be proved inductively using perturbation theory. In Sect. 3 we show that the decay estimate on $G$ and (1.6) imply Theorems 1.1 and 1.2.

The above inequality imposes a special geometric pattern on the intervals $I_{n}^{i} \in S_{n}$ as can be seen from the following lemma:

Lemma 1.3. Let $a, b, c \in \mathbb{Z}$, be distinct points. If $m(a, b) \leq \delta$ and $m(b, c) \leq \delta$, then either $|a-b| \geq \delta^{-1 / 2} C_{1} / 3$ or $|b-c| \geq \delta^{-1 / 2} C_{1} / 3$. Also if $|a-b| \leq \delta^{-1 / 2} C_{1} / 5$, then there is a unique $\tilde{c}$ such that $|a-b|=|c-\tilde{c}|$ and $m(c, \tilde{c})=\left|(c+\tilde{c}) \alpha+2 \theta_{*}\right|_{1} \leq 3 \delta$.

Proof. If $|(i-j) \alpha|_{1} \leq \delta$, then by (1.4), $|i-j| \geq \delta^{-1 / 2} C_{1}$. Thus we need only consider the case where $\left|(a+b) \alpha+2 \theta_{*}\right|_{1} \leq \delta$ and $\left|(b+c) \alpha+2 \theta_{*}\right|_{1} \leq \delta$. From these inequalities we deduce $|(a-c) \alpha|_{1} \leq 2 \delta$ so $|a-c| \geq \delta^{-1 / 2} C_{1} / \sqrt{2}$, and our first assertion follows easily. To prove the next assertion we can assume $\left|(a+b) \alpha+2 \theta_{*}\right|_{1} \leq \delta$; otherwise $|(a-b) \alpha|_{1} \leq \delta$, which by (1.4) contradicts our distance assumption. If $|(b-c) \alpha|_{1} \leq \delta$, then $m(\tilde{c}, c) \leq\left|[(c+a-b) \alpha+c \alpha]+2 \theta_{*}\right|_{1} \leq$ $|(a+b) \alpha+2 \theta *|_{1}+2|(b-c) \alpha|_{1} \leq 3 \delta$, where $\tilde{c}=c+(a-b)$. If $\left|(b+c) \alpha+2 \theta_{*}\right|_{1} \leq \delta$, then we set $\tilde{c}=c-(a-b)$ and a similar inequality holds. The uniqueness of $\tilde{c}$ follows from the first part of the lemma.

We call $\tilde{c}$ the "mirror image" of $c$, because, for all $j, v\left(-j+\tilde{c}, \theta_{*}\right)=v(j+c$, $\left.\theta_{*}\right)+\mathcal{O}(\delta)$. This lemma together with (1.6) shows that each interval $I_{n}$ of width $l_{n}$ can contain no more than two intervals $I_{n-1}$. A third interval is excluded, since Const. $l_{n} \leq C_{1} \delta_{n-1}^{-1 / 4}$. 
For the lattice, $S_{0}$ has a very simple description. Intervals consist of single sites, $E_{0}^{i}=v\left(i, \theta_{*}\right)$ and

$$
S_{0}\left(E_{*}, \theta_{*}\right)=\left\{j:\left|v\left(j, \theta_{*}\right)-E_{*}\right| \leq \delta_{0}\right\},
$$

where $\delta_{0}=\varepsilon$. Since $|v(a, \theta *)-v(b, \theta *)| \geq m(a, b)^{2} / 2$, (1.6) holds for $n=0$. Also note that if $\Lambda$ is an interval such that $\Lambda \cap S_{0}=\emptyset$, then since $\|\Delta\|=2$, and $\Delta$ has only nearest neighbor matrix elements,

$$
\begin{aligned}
\left|G_{\Lambda}\left(E_{*} ; x, y\right)\right|= & \sum_{n=0}^{\infty}\left[\left|v(j)-E_{*}\right|^{-1} \varepsilon^{2} \Delta\right]^{n}(x, y)\left|v(y)-E_{*}\right|^{-1} \\
& \leq \frac{1}{2} \sum_{n \geq|x-y|} \varepsilon^{n-1} \leq \varepsilon^{|x-y|-1}
\end{aligned}
$$

In the case of $H_{c}$ we define $I_{0}^{i}$ to be intervals of width 1 centered at $c_{0}^{i} \in \mathbb{Z}$. Let $\left|E_{*}+2 K^{2}\right| \leq 10 K$ and define

$$
S_{0}=\left\{I_{0}^{i}: \inf _{x \in I_{0}^{i}}\left[K^{2} v(x)-E_{*}\right] \leq 2 K\right\}
$$

It is easy to se that these intervals are centered at near minima of $v(x)$ and that they are widely separated $\cong K^{1 / 4}$. Notice that if $\Lambda \cap S_{0}=\emptyset$ then $\left|G_{\Lambda}\left(E_{*}, x, y\right)\right| \leq$ $\exp \left[-\gamma_{0}|x-y|\right]$, where $\gamma_{0}=(2 K)^{1 / 2}$. This follows from the maximum principle. (The constant $\gamma_{0}$ can be improved to $K /$ const by using a simple W.K.B. estimate.) For $n=0,(1.6)$ holds with $\delta_{0}=K^{-1}$. See Appendix C for further discussion of the $n=0$ case.

It is important to note that since $I_{m}^{i}$ and $I_{m}^{j}$ are integral translates of each other the spectrum of $H, \sigma H$, satisfies

$$
\sigma H\left[I_{n}^{i}, \theta_{*}\right]=\sigma H\left[I_{n}^{j}, \theta_{*}+\left(c_{n}^{i}-c_{n}^{j}\right) \alpha\right]=\sigma H\left[I_{n}^{j}, \theta_{*}-\left(2 \theta_{*}+\left(c_{n}^{i}+c_{n}^{j}\right) \alpha\right)\right] .
$$

The same identity holds for $H_{c}$. In the second equality we have used the evenness of the cosine: $v\left(x+c_{n}^{j}, \theta_{*}\right)=v\left(-x+c_{n}^{j},-\theta_{*}-2 c_{n}^{j} \alpha\right)$. Since we are in one dimension, the eigenvalues as functions of $\theta$ never cross and are smooth in $\theta$. Hence the evenness implies

$$
E_{m}^{j}(\theta)=E_{m}^{j}\left(-\theta-2 c_{m}^{j} \alpha\right) .
$$

To prove (1.6), we shall show that (1.7) holds for the particular eigenvalues $E_{n+1}^{i}(\theta)$ i.e. $E_{n+1}^{i}\left(\theta_{*}\right)=E_{n+1}^{j}\left(\theta_{*} \pm m\left(c_{n+1}^{i}, c_{n+1}^{j}\right)\right)$. Our key estimate gives a bound from below, $\left|d^{2} E_{n+1}^{i}(\theta) / d \theta^{2}\right| \geq 2 / 3$ when $\left|d E_{n+1}^{i}(\theta) / d \theta\right|$ is small. Hence if $E_{n+1}^{i}(\theta)$, $E_{n+1}^{j}(\theta)$ satisfy (1.5) then we obtain (1.6) in the following form

$$
2 \delta_{n+1} \geq\left|E_{n+1}^{i}\left(\theta_{*}\right)-E_{n+1}^{j}\left(\theta_{*}\right)\right|=\left|E_{n+1}^{i}\left(\theta_{*}\right)-E_{n+1}^{i}\left(\theta_{*}+m\right)\right| \geq \frac{m^{2}}{4},
$$

where $m= \pm m\left(c_{n+1}^{i}, c_{n+1}^{j}\right)$. The last inequality of (1.9) follows from the lower bound on $\left|d^{2} E / d \theta^{2}\right|$ and an elementary lemma, Lemma 5.7. See Sect. 5, for details.

The outline of this paper is as follows. Most of the paper is devoted to discussing the finite difference operator $H$ with occasional references to what needs to be modified for the analysis of $H_{c}$. In the next section we give a precise 
definition of $S_{n}$ and present key identities we shall need for our analysis. The proof of Theorems 1.1 and 1.2 are given in Sect.3, assuming that (1.6) holds for all $n$, and that the Green's function decays. In Sect. 4 we assume (1.6) for fixed $n$ and establish properties of the eigenvalues $E_{n+1}$ and the corresponding eigenfunctions. In Sect. 5 we also assume (1.6) and establish a lower bound on $\left|d^{2} E_{n+1}^{i}(\theta) / d \theta^{2}\right|$ when $\left|d E_{n+1}^{i}(\theta) / d \theta\right|$ is small. This enables us to prove (1.6) at the next scale, $n+1$, as in (1.9). Some technical comments about the continuum case appear in Appendix C.

\section{Definitions and Formulas}

Let $E_{*}, \theta_{*}$ be fixed. For $n=0, S_{0}$ is defined in Sect. 1 and (1.6) holds. Let $n \geq 0$. Now assume that $S_{n}$ is defined and that we have proved by induction that (1.6) holds. We define $S_{n+1}=S_{n+1}\left(E_{*}, \theta_{*}\right)$ as follows. Let

$$
s_{n}=\min \left\{\left|c_{n}^{i}-c_{n}^{j}\right|: I_{n}^{i}, I_{n}^{j} \in S_{n}\right\} .
$$

The $c_{n}^{i}$ denote the centers of $I_{n}^{i}$. For $n \geq 1$, if $s_{n} \geq 4 l_{n}^{2}$, let $I_{n+1}^{i}$ have length $l_{n+1} \cong l_{n}^{2}$ and center $c_{n+1}^{i}=c_{n}^{i}$. In this case $I_{n+1}^{i}$ contains a single interval of $S_{n}$.

If $s_{n} \leq 4 l_{n}^{2}$ we note that each interval $I_{n}^{i} \in S_{n}$ has a "mirror" image $\tilde{I}_{n}^{i}$ whose center satisfies $\left|c_{n}^{i}-\tilde{c}_{n}^{i}\right|=s_{n}$ and

$$
m\left(c_{n}^{i}, \tilde{c}_{n}^{i}\right)=\left|2 \theta_{*}+\left(c_{n}^{i}+\tilde{c}_{n}^{i}\right) \alpha\right|_{1} \leq 12 \delta_{n}^{1 / 2} .
$$

This follows from (1.6) and Lemma 1.3. These considerations also imply that the center of any other interval $I_{n}^{j} \in S_{n}$ satisfies

$$
\left|c_{n}^{j}-c_{n}^{i}\right| \geq C_{1} \delta_{n}^{-1 / 4} / 12 \geq l_{n+2} \geq l_{n+1} .
$$

The intervals $I_{n+1}^{i}$ are now defined to be centered at $c_{n+1}^{i}$ and of width $l_{n+1}$, where

$$
c_{n+1}^{i}=\frac{1}{2}\left[c_{n}^{i}+\tilde{c}_{n}^{i}\right], \quad l_{n+1} \cong l_{n}^{4} .
$$

The collection of intervals consisting of $S_{n}$ and of its mirror image is called $\bar{S}_{n}$. Note that in the present case $I_{n+1}^{i}$ contains precisely 2 elements of $\bar{S}_{n}$. By (2.3) $I_{n+1}^{i}$ do not overlap (see Fig. 1).

a

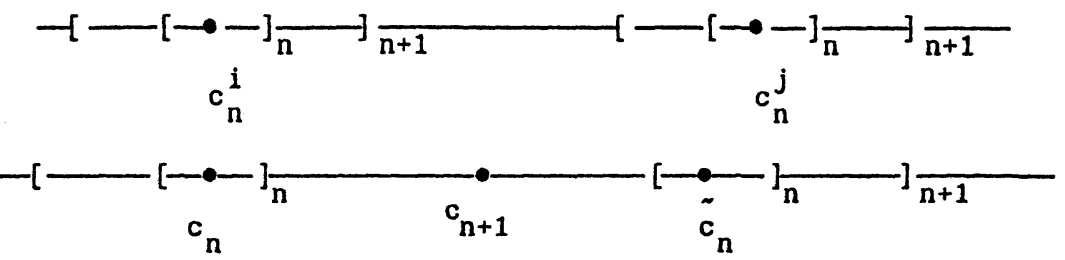

Fig. 1. a The intervals $I_{n}^{i}$ and $I_{n}^{j}$ are separated by more than $4 l_{n}^{2}$. The $\bullet$ denote the centers of the intervals $I_{n}^{i}, I_{n}^{i}$. In this case $c_{n+1}^{i}=c_{n}^{i}$ and $c_{n+1}^{j}=c_{n}^{j}$. b The interval $I_{n}$ and $\tilde{I}_{n}$ are separated by less than $4 l_{n}^{2}$. The interval $I_{n+1}$ is much larger than indicated in the figure

For $n=0$, we define $S_{1}$ as above except that $l_{0}^{2}$ and $l_{0}^{4}$ above are replaced by $L^{2}, L^{4}$ where $L=|\ln \varepsilon|$. This is because $l_{0}$ is small and we want $l_{1}$ to be large. This special convention will be adopted in the remainder of this paper. 
Definition. An interval $I_{n+1}^{i}$ belongs to the family of singular intervals $S_{n+1}\left(E_{*}, \theta_{*}\right)$ if $H\left(I_{n+1}^{i}\right)$ has an eigenvalue $E_{n+1}^{i}\left(\theta_{*}\right)$ such that

$$
\left|E_{*}-E_{n+1}^{i}\right| \leq \delta_{n+1} \equiv \exp \left(-\beta l_{n+1}^{2 / 3}\right),
$$

where $\beta=|\ln \varepsilon|$. The nonsingular intervals $I_{n+1}$ are called $(n+1)$-regular. The length $l_{n+1}$ of the intervals $I_{n+1}$ is chosen so that its boundary, $\partial I_{n+1}$, does not meet $I_{m}^{i}$ for $m \leq n$. This can be done using Lemma 2.4 below. An arbitrary interval $\Lambda$ is said to be $n$-regular if every point (or interval) of $\Lambda \cap S_{0}$ is contained in an $m$ regular interval $I_{m} \subset \Lambda$ for some $m \leq n$.

In the continuum, the definitions are as above except $\beta=K / 2, \delta_{0}=K^{-1}$. Later we shall use the notation $\delta_{-1}=\varepsilon^{1 / 4}, K^{1 / 4}$ for the discrete and continuum cases respectively.

Next we formulate condition $\mathscr{C}_{n}$ on the centers of the singular intervals.

Condition $\mathscr{C}_{n}$. For all $m \leq n$ whenever $c_{m}^{i}, c_{m}^{j}$ are centers of intervals $I_{m}^{i}, I_{m}^{j} \in S_{m}$ then

$$
m\left(c_{m}^{i}, c_{m}^{j}\right)^{2} \leq 8\left|E_{m}^{i}-E_{m}^{j}\right| \leq 16 \delta_{m} .
$$

Note that (2.6) is a stronger version of (1.6).

Theorem 2.1. If $\varepsilon$ is small $\mathscr{C}_{n}$ holds for all $n, \theta_{*}$ and $E_{*}$.

The following remarks hold for all $m \leq n$, assuming $\mathscr{C}_{n}$ holds.

Remarks. a) The last inequality in (2.6) follows from the fact that both $E_{m}^{i}$ and $E_{m}^{j}$ belong to the interval $\left|E-E_{*}\right| \leq \delta_{m}$.

b) If $s_{m} \geq 4 l_{m}^{2}$, then $I_{m+1}^{j}$ contains precisely one interval $I_{m}^{j}$ in $S_{m}$ and $I_{m+1}^{j} \backslash I_{m}^{j}$ is the union of $m$-regular intervals.

c) If $s_{m} \leq 4 l_{m}^{2}$, then $I_{m+1}^{i}$ contains exactly two intervals in $\bar{S}_{m}, I_{m}, \tilde{I}_{m}$. By (1.7) and (2.2) the spectrum of $H\left(I_{m}\right)$ and $H\left(\tilde{I}_{m}\right)$ differ by $\mathcal{O}\left(\delta_{m}^{1 / 2}\right)$.

d) If $s_{m-1} \leq 4 l_{m-1}^{2}$ then, by (2.3) the pairs of intervals in $\bar{S}_{m-1}$ are so far separated that $s_{m} \geq 4 l_{m}^{2}$ and $s_{m+1} \geq 4 l_{m+1}^{2}$.

Theorem 2.2. For $\varepsilon^{2}$ sufficiently small (independent of $n$ ) if $\mathscr{C}_{n-1}$ holds and $\Lambda$ is n-regular then

$$
\left|G_{\Lambda}(E, x, y)\right| \leq \exp -\gamma_{n}|x-y|
$$

for all $\left|E-E_{*}\right| \leq \delta_{n} / 3,\left|\theta-\theta_{*}\right| \leq \delta_{n} / 3$ provided $|x-y| \geq l_{n}^{5 / 6}$. Moreover $\gamma_{n} \geq$ $\gamma \geq \frac{1}{2} \mid \ln \varepsilon$.

For notational compactness we shall generally omit the $\theta$ dependence of $G$.

Analogous theorems hold in the continuum:

Theorem 2.3. If $K$ is sufficiently large and $E_{*} \in\left[-2 K^{2},-2 K^{2}+10 K \sqrt{1+\alpha^{2}}\right]$, then $\mathscr{C}_{n}$ holds for all $n$ and $\theta *$. For large $K$ (2.7) holds as above if we set $\gamma_{n} \geq$ $\gamma \geq K /$ const and require $\left|\theta-\theta_{*}\right| \leq \delta_{n} / 16 \pi K^{2}$. Derivatives of $G$ in $x, y \in R$ also satisfy (2.7). See (2.9) below.

The proof of Theorem 2.2 is given in $[10,12]$. See Appendix A where the key steps are explained. Theorem 2.1 will be proved by induction on $n$. 
In the proof of the above theorems we shall frequently express $G_{\Lambda}=[H(\Lambda)-$ $E]^{-1}$ in terms of $G_{I}$ where $I$ is a subinterval of $\Lambda$. Let us define

$$
G_{\Lambda, I}=G_{\Lambda \backslash I} \oplus G_{I} \text {. }
$$

On the lattice, we can write

$$
H(\Lambda)=H(I)+H(\Lambda \backslash I)-\varepsilon^{2} \Gamma,
$$

so by the resolvent identity

$$
G_{\Lambda}=G_{\Lambda, I}+\varepsilon^{2} G_{\Lambda, I} G_{\Lambda} .
$$

Here $\Gamma$ denotes the symmetric matrix corresponding to the boundary of $I$ in $A$

$$
\begin{aligned}
\Gamma_{x, y} & =1 & & \text { if } \quad(x, y) \in \partial I \\
& =0 & & \text { otherwise. }
\end{aligned}
$$

On the lattice we have represent $\partial I$ as a set of unordered nearest neighbor pairs $\left(a, a^{\prime}\right)$ such that $a \in I, a^{\prime} \notin I$. We shall frequently identify the boundary of a set with the corresponding matrix, as above. The continuum analogue of $(2.8)$ is

$$
G_{\Lambda}(x, y)=G_{\Lambda, I}(x, y)+\sum_{z \in \partial I \backslash \partial \Lambda} \dot{G}_{\Lambda, I}(x, z) G_{\Lambda}(z, y),
$$

where $\dot{G}$ denotes the normal derivative of $G$ with respect to $z$.

The key estimates involve derivatives of $E_{n}^{i}(\theta)$ with respect to $\theta$. Note that, since we are in one dimension, there is no level crossing, and $E_{n}^{i}(\theta)$ is well defined and smooth in $\theta$. The first and second derivatives of an eigenvalue $E(\theta)$ of $H(I)$ are given by first and second order perturbation theory:

$$
\begin{gathered}
\frac{d}{d \theta} E(\theta)=\left\langle\psi, v^{\prime} \psi\right\rangle \quad v^{\prime}=\frac{d v}{d \theta}, \\
\frac{d^{2}}{d \theta^{2}} E(\theta)=\left\langle\psi, v^{\prime \prime} \psi\right\rangle-2\left\langle\psi, v^{\prime} G_{I}^{\perp}(E) v^{\prime} \psi\right\rangle,
\end{gathered}
$$

where $\psi$ is the normalized eigenfunction corresponding to $E(\theta)$, and $G_{I}^{\perp}(E)$ is the Green's function projected onto the orthogonal complement of $\psi$. If $\tilde{E}$ denotes the eigenvalue of $H(I)$ closest to $E$, and $\tilde{\psi}$ its eigenfunction, then after projecting out $\tilde{\psi}$ we have

$$
\left\langle\psi, v^{\prime} G_{I}^{\perp}(E) v^{\prime} \psi\right\rangle=\frac{\left\langle\psi, v^{\prime} \tilde{\psi}\right\rangle^{2}}{\tilde{E}-E}+\left\langle\psi, v^{\prime} G_{I}^{\perp \perp}(E) v^{\prime} \psi\right\rangle
$$

The same formulas hold for $H_{c}$, except $v$ is replaced by $K^{2} v$. In Sect. 5 we show that when $d E / d \theta$ is small, $\left|\left\langle\psi, v^{\prime} \tilde{\psi}\right\rangle\right|$ is bounded below and $|E-\tilde{E}|$ is small: hence by (2.10) and (2.11), $\left|E^{\prime \prime}(\theta)\right|$ is large. This is the key step in our proof which enables us to justify (1.9) and (2.6).

Using (2.10) it is easy to see that for any eigenvalue $E(\theta)$ of $H(I, \theta)$,

$$
|d E(\theta) / d \theta| \leq 1, \quad|d E(\theta) / d \theta| \leq 4 \pi K^{2}
$$

for the discrete and continuum cases respectively. This will be used to obtain narrow intervals in $\theta$ near $\theta *$ where our theorems and lemmas apply. 
We conclude this section with a technical lemma, assuming $\mathscr{C}_{n}$ holds.

Lemma 2.4. If $\Lambda=[a, b]$ is an interval then there is a deformed interval $\Lambda^{\prime}=$ $\left[a^{\prime}, b^{\prime}\right]$ such that $a^{\prime}, b^{\prime} \notin I_{m}^{i}$, for all $m \leq n$, and $\left|a-a^{\prime}\right|+\left|b-b^{\prime}\right| \leq 3 l_{n}$.

Proof. By construction the intervals $I_{n}^{i}$ are separated by a distance of at least $2 l_{n}$. The endpoint $b$ can be moved to $b_{1}$ by a distance of less than $l_{n}$ so that $\operatorname{dist}\left(b_{1}, I_{n}^{i}\right) \geq l_{n} / 2$ for all $i$. Similarly $b_{1}$ can be moved to $b_{2}$, by a distance of less than $l_{n-1}$, so that $\operatorname{dist}\left(b_{2}, I_{n-1}^{i} \geq l_{n-1} / 2\right.$, etc. By using the fact that $l_{n} / 2 \geq \sum_{m=0}^{n-1} l_{m}$ we see that $b^{\prime} \equiv b_{n}$ belongs to no $I_{m}^{i}, m \leq n$, and $\left|b-b^{\prime}\right| \leq 3 l_{n} / 2$. The other endpoint, $a$, is treated similarly.

This lemma enables us to adjust the approximate length of $I_{n+1}^{i}$, which is $l_{n}^{2}$ or $l_{n}^{4}$, by $\mathcal{O}\left(l_{n}\right)$ so that the endpoints do not meet $I_{m}^{j} \in S_{m}$, for all $m \leq n$. See Appendix D of [10]. This is a requirement of our definition of $S_{n+1}$. Furthermore, using the special "selfsimilar" structure of $S_{m}$ or $\bar{S}_{m}$ described in Lemma 1.3, we can choose $l_{n+1}$ independent of $i$. This is convenient because otherwise equalities relating $E_{n}^{i}$ to $E_{n}^{j}$ have exponentially small corrections.

\section{Pure Point Spectrum}

We shall follow the strategy of [11] to prove that $H$ has pure point spectrum. By a theorem of Berezanskii $[13,14]$ it is sufficient to prove that every generalized eigenfunction decays exponentially fast. By a generalized eigenfunction we mean any nonzero solution to the equation $H(\theta) \psi=E(\theta) \psi$ such that $|\psi(j)| \leq$ const. $|j|^{2}$.

Let $E(\theta)$ be a generalized eigenvalue and let $\Lambda_{n}$ be an interval of length $2 l_{n}$ centered at 0 .

Lemma 3.1. There is an $N=N(\theta, E(\theta))$ such that for all $n \geq N, \Lambda_{n} \cap I_{n}^{i} \neq \emptyset$ for some $I_{n}^{i} \in S_{n}(E(\theta), \theta)$.

Proof. If not, there is a sequence $s=n_{i} \rightarrow \infty$ such that $\Lambda_{s} \cap I_{s}^{j}=\emptyset$ for all $I_{s}^{j} \in S_{s}(E)$. Now using Lemma 2.4, choose $\Lambda_{s}^{\prime}$ to be $s$-regular intervals such that $0 \in \Lambda_{s}^{\prime} \subset \Lambda_{s}$ and $\operatorname{dist}\left(0, \partial \Lambda_{s}^{\prime}\right) \geq l_{s} / 2$. If $H(\theta) \psi=E(\theta) \psi$, then $\psi(j)$ can be determined from its values on $\partial \Lambda_{s}^{\prime}$. For $j \in \Lambda_{s}^{\prime}$,

$$
|\psi(j)|=\varepsilon^{2}\left|\sum_{k, l} G^{\prime}(E(\theta), j, k) \Gamma_{k l}^{\prime} \psi(l)\right| .
$$

Here $G^{\prime}$ is the Green's function of $H\left(\Lambda_{s}^{\prime}\right)$ and $\Gamma^{\prime}=\partial \Lambda_{s}^{\prime}$. For $|j| \leq l_{s} / 4$ we see that $|j-k| \geq l_{s} / 4$, so that by Theorem 2.2 and the subquadratic growth of $\psi$,

$$
|\psi(j)| \leq 2 e^{-\gamma l_{s} / 4} l_{2}^{2} \varepsilon^{2}
$$

Since $\gamma \geq 1$ and $l_{s}$ can be arbitrarily large $\psi(j) \equiv 0$. This contradiction proves the lemma. 
Lemma 3.2. For almost all $\theta_{*}$ and any generalized eigenvalue $E\left(\theta_{*}\right)$, there is a finite $N=N\left(\theta_{*}, E\left(\theta_{*}\right)\right)$ such that for all $n \geq N, \bar{S}_{n}\left(E\left(\theta_{*}\right), \theta_{*}\right)$ has a unique interval $I_{n}^{*} \subset \Lambda_{n+2}$. These intervals have a common center $c_{N}^{*}$ and $\left|c_{N}^{*}\right| \leq 3 l_{N} / 2$.

Proof. The existence of the interval $I_{n}^{*}$ follows from Lemma 3.1. If there are two intervals $I_{n}^{*}, I_{n}^{\prime} \subset A_{n+2}$, with $I_{n}^{\prime} \in \bar{S}_{n}$, then by Theorem 2.1 and (2.2), the corresponding centers satisfy $m\left(c_{n}^{*}, c_{n}^{\prime}\right) \leq 12 \delta_{n}^{1 / 2}$. Since $\left|c_{n}^{*}-c_{n}^{\prime}\right| \leq c l_{n}^{8}$ the Diophantine condition (1.4) implies that

$$
m\left(c_{n}^{*}, c_{n}^{\prime}\right)=\left|\left(c_{n}^{*}+c_{n}^{\prime}\right) \alpha+2 \theta *\right|_{1} \leq 12 \delta_{n}^{1 / 2} .
$$

The set of $\theta_{*}$ for which (3.2) holds has measure less than $6 \delta_{n}^{1 / 2}$ and is independent of $E\left(\theta_{*}\right)$. Since the number of possible pairs of centers in $\Lambda_{n+2}$ is less than $4 l_{n+2}^{2}$ the probability that $\Lambda_{n+2}$ contains 2 singular intervals is less than Const. $\delta_{n}^{1 / 2} l_{n+2}^{2}$. Since the sum over $n$ is finite, by the Borel-Cantelli lemma, with probability one with respect to $\theta_{*}$, there is an $N<\infty$ so that $I_{n}^{*}$ is unique for all $n \geq N$ and so $c_{n}^{*}=c_{N}^{*}$ all $n \geq N$ by construction.

Proof of Theorem 1.1. Let $E\left(\theta_{*}\right)$ be a generalized eigenvalue and $N\left(\theta_{*}\right)$ as in the previous lemma. Let $|x| \geq l_{N+1}$. We claim that there is an interval $A$ containing $x$ such that for some $n$,

a) $A$ is $n$ regular,

b) $\operatorname{dist}(\partial \Lambda, x) \geq|x| / 3 \geq l_{n} / 2$.

Therefore by Theorem 2.2 and the polynomial bound on $\psi$

$$
|\psi(x)|=\left|\sum_{z, z^{\prime}} G_{\Lambda}(E ; x, z) \Gamma_{z z^{\prime}} \psi\left(z^{\prime}\right)\right| \varepsilon^{2} \leq \exp \left(-\frac{\gamma|x|}{3}\right),
$$

where $\Gamma=\partial \Lambda$.

To establish our claim, let $n \geq N+1$ be defined so that $l_{n+1} \geq|x| \geq l_{n}$. The interval $\Lambda^{\prime}=\left[\frac{x}{2}, \frac{3 x}{2}\right]$ does not meet $I_{n-1}^{*}$ because $\left|c_{n-1}^{*}\right|=\left|c_{N}^{*}\right| \leq 2 l_{N}$. By Lemma 3.2, since $\Lambda^{\prime} \subset \Lambda_{n+1}$, we see that $\Lambda^{\prime}$ meets no member of $S_{n-1}$. Using Lemma 2.4, we can deform the interval $\Lambda^{\prime}$ to $\Lambda$ so that it is $n$-regular and b) holds.

The proof of Theorem 1.2 is exactly the same, except that we use Theorem 2.3 and the formula

$$
\psi(x)=\sum_{z \in \partial \Lambda} \dot{G}_{\Lambda}(E, x, z) \psi(z),
$$

to recover $\psi$ from its values on $\partial \Lambda$. Recall $\dot{G}$ is the normal derivative of $G$.

\section{Eigenfunctions of $H\left(I_{n+1}\right)$}

In this section we establish some basic facts about the eigenvalues and eigenfunctions of $H\left(I_{n+1}\right)$ for $I_{n+1} \in S_{n+1}$. We shall assume that $\mathscr{C}_{n}$ holds [see (2.6)]. If $I_{n+1}$ contains a single element $I_{n} \in S_{n}$ (i.e. $s_{n} \geq 4 l_{n}^{2}$ ) then we show that there is only one relevant eigenvalue $E_{n+1}$ near $E_{*}$ and the eigenfunction is localized well inside $I_{n}$. If $I_{n+1}$ contains two singular intervals (i.e. $s_{n} \leq 4 l_{n}^{2}$ ) $I_{n}^{+}$and $I_{n}^{-} \in \bar{S}_{n}$, then 
there are two relevant eigenvalues $E_{n+1}$ and $\tilde{E}_{n+1}$. The eigenfunctions are well localized in $I_{n}^{+} \cup I_{n}^{-}$. We use the one dimensionality of space to ensure that there are no level crossings, so $E_{n}(\theta)$ is a smooth function of $\theta$. The following lemma can be used to bound differences of eigenvalues. In more than one dimension these differences can be obtained by induction (see [9]).

Lemma 4.1. Let $\psi_{1}$ and $\psi_{2}$ be eigenfunctions of $H(\Lambda)$, where $\Lambda$ is an interval. If there is an $a \in \mathbb{Z}$ such that

$$
\left\|[1-x(|x-a| \leq l)] \psi_{i}\right\| \leq \frac{1}{2}\left\|\psi_{i}\right\|, \quad i=1,2,
$$

then the corresponding eigenvalues satisfy

$$
\begin{aligned}
& \left|E_{1}-E_{2}\right| \geq\left(\frac{\varepsilon}{2}\right)^{4(l+1)} \quad \text { (finite difference), } \\
& \left|E_{1}-E_{2}\right| \geq e^{-(4 K+1) l} \quad \text { (continuum) } .
\end{aligned}
$$

The proof of this proposition is standard (see [15]). A proof is given in Appendix B.

Proposition 4.2. If $I_{n+1} \in \bar{S}_{n+1}$ and $s_{n} \geq 4 l_{n}^{2}$, then $H\left(I_{n+1}\right)$ has a unique eigenvalue $E_{n+1}$ in the interval $\left|E-E_{*}\right| \leq \delta_{n} / 3$ for $\left|\theta-\theta_{*}\right| \leq \delta_{n} / 3$ and the corresponding eigenfunction satisfies

$$
|\psi(x)| \leq e^{-\gamma\left|c_{n}-x\right| / 3}
$$

for $\left|x-c_{n}\right| \geq 4 l_{n}^{1 / 2}$. Moreover $\left\|G_{n+1}^{\perp}\left(E_{n+1}\right)\right\| \leq 3 \delta_{n}^{-1}$, where $G_{n+1}^{\perp}$ denotes the Green's function for $I_{n+1}$ on the orthogonal complement of $\psi_{n+1}$.

Proof. We first suppose that $s_{n} \geq 4 l_{n}^{2}$ and $s_{n-1} \geq 4 l_{n-1}^{2}$. Then $I_{n+1} \backslash I_{n}$ and $I_{n} \backslash I_{n-1}$ are respectively $n$ and (n-1)-regular, hence $\Lambda \equiv I_{n+1} \backslash I_{n-1}$ is $n$ regular. For $n=0$ let $\Lambda \equiv I_{1} \backslash\left\{c_{0}\right\}$. Let $E \in \sigma H\left(I_{n+1}\right)$ be such that $\left|E-E_{*}\right| \leq \delta_{n} / 3$. Let $\left|x-c_{n}\right| \geq l_{n} / 2$ and $\Gamma=\partial I_{n-1}$. We determine the value of $\psi(x)$ from its values on the boundary of $I_{n-1}$ :

$$
\psi(x)=\varepsilon^{2} \sum_{y, y^{\prime}} G_{\Lambda}(E, x, y) \Gamma_{y y^{\prime}} \psi\left(y^{\prime}\right) .
$$

The decay of $\psi,(4.3)$, now follows from Theorem 2.2 and the inequality

$$
|x-y| \geq\left|x-c_{n}\right|-\left|c_{n}-y\right| \geq\left|x-c_{n}\right|-l_{n-1} \geq \frac{3}{4}\left|x-c_{n}\right| \geq l_{n}^{5 / 6} \text {. }
$$

To obtain decay for $l_{n} / 2 \geq\left|x-c_{n-1}\right| \geq 2 l_{n-1}=2 l_{n}^{1 / 2}$ we set $A=I_{n} \backslash I_{n-1}$ which is $(n-1)$-regular and apply (4.4) and Theorem 2.2 as before. Note that $c_{n}=c_{n-1}$.

Next we suppose $s_{n-1} \leq 4 l_{n-1}^{2}$. Then $I_{n} \subset I_{n+1}$ contains precisely two intervals $I_{n-1}^{+}, I_{n-1}^{-}$in $\bar{S}_{n-1}$. Set $\Lambda=I_{n+1} \backslash\left(I_{n-1}^{+} \cup I_{n-1}^{-}\right)$. $\Lambda$ is $(n-1)$-regular, because $(2.3)$ shows that $I_{n+1}$ cannot contain three $(n-1)$-singular intervals. Let $\left|E-E_{*}\right| \leq$ $\delta_{n-1} / 3$ and set $\Gamma=\partial I_{n-1}^{+} \cup \partial I_{n-1}^{-}$. Then again applying Theorem 2.2 to (4.4) one obtains

$$
\begin{aligned}
|\psi(x)| & \leq \exp \left(\frac{-3 \gamma}{4}\left|c_{n-1}^{+}-x\right|\right)+\exp \left(\frac{-3 \gamma}{4}\left|c_{n-1}^{-}-x\right|\right) \\
& \leq e^{-\gamma\left|c_{n}-x\right| / 3}
\end{aligned}
$$


for $\left|c_{n}-x\right| \geq 4 l_{n}^{1 / 2} \cong 4 l_{n-1}^{2}$. In the last equality we used $c_{n}=\left(c_{n-1}^{+}+c_{n-1}^{-}\right) / 2$ and $\left|c_{n-1}^{+}-c_{n-1}^{-}\right| \leq 4 l_{n-1}^{2}$.

Thus any eigenvalue in the interval $\left|E-E_{*}\right| \leq \delta_{n} / 3$ corresponds to an eigenfunction localized in an interval of width less than $4 l_{n}^{1 / 2}$. If $I_{n+1} \in S_{n+1}$, then by definition there is an eigenvalue $E_{n+1}$ such that $\left|E_{n+1}-E_{*}\right| \leq \delta_{n+1}$. By Lemma 4.1 it is unique since any other eigenvalue $\hat{E}$ satisfies

$$
\left|\hat{E}-E_{n+1}\right| \geq \varepsilon^{4\left(l_{n}^{1 / 2}+1\right)} \geq \delta_{n}=e^{-\beta l_{n}^{2 / 3}} .
$$

Our bound on $G^{\perp}\left(E_{n+1}\right)$ follows easily from this estimate. If $\tilde{I}_{n+1}$ is the "mirror" image of $I_{n+1} \in S_{n+1}$, then by (2.2), $m\left(\tilde{c}_{n+1}, c_{n+1}\right)=\mathcal{O}\left(\delta_{n+1}^{1 / 2}\right)$, and so by (1.7) and (2.13) the spectrum of $H\left(I_{n+1}^{+}\right.$and $H\left(I_{n+1}^{-}\right)$differ only $\mathcal{O}\left(\delta_{n+1}^{1 / 2}\right)$. Therefore the same argument applies for $I_{n+1} \in \bar{S}_{n+1}$.

Next we consider the case where $s_{n} \leq 4 l_{n}^{2}$. Then each $I_{n+1} \in S_{n+1}$ contains two intervals $I_{n}^{+}, I_{n}^{-} \in \bar{S}_{n}$ and $s_{n-1} \geq 4 l_{n-1}^{2}$ and $s_{n-2} \geq 4 l_{n-2}^{2}$. See the remarks following Theorem 2.1.

Proposition 4.3. If $I_{n+1} \in S_{n+1}$ and $s_{n} \leq 4 l_{n}^{2}$, then $H\left(I_{n+1}\right)$ has exactly two eigenvalues $E_{n+1}, \tilde{E}_{n+1}$ in the interval $\left|E-E_{*}\right| \leq \delta_{n-1} / 3$ for $\left|\theta-\theta_{*}\right| \leq \delta_{n}^{1 / 2}$. The eigenfunctions satisfy

$$
\begin{aligned}
& \psi_{n+1}=A \psi_{n}^{+}+B \psi_{n}^{-}+\mathcal{O}\left(\delta_{n}^{3}\right), \\
& \tilde{\psi}_{n+1}=B \psi_{n}^{+}-A \psi_{n}^{-}+\mathcal{O}\left(\delta_{n}^{3}\right),
\end{aligned}
$$

where $A^{2}+B^{2}=1$ and $\psi_{n}^{+}$and $\psi_{n}^{-}$are the normalized eigenfunctions of $H\left(I_{n}^{+}\right)$and $H\left(I_{n}^{-}\right)$respectively whose eigenvalues $E_{n}^{+}, E_{n}^{-}$are closest to $E_{*}$. Also $\left\|G_{n+1}^{\perp \perp}\left(E_{n+1}\right)\right\|$ $\leq \mathcal{O}\left(\delta_{n-1}^{-1}\right)$.

Proof. For $n \geq 1$, the intervals $I_{n}^{+}$and $I_{n}^{-}$contain just one singular interval each, $I_{n-1}^{+}, I_{n-1}^{-}$. As explained in the previous lemma $\Lambda=I_{n+1} \backslash\left(I_{n-1}^{+} \cup I_{n-1}^{-}\right)$is $(n-1)$-regular and for $E \in H\left(I_{n+1}\right),\left|E-E_{*}\right| \leq \delta_{n-1} / 3$, and $\left|\theta-\theta_{*}\right| \leq \delta_{n-1} / 3$, we can conclude that the corresponding eigenfunction $\psi$ decays exponentially fast for $x$ away from $c_{n-1}^{+}, c_{n-1}^{-}$as in (4.5).

To establish (4.6), first note that for $j \in I_{n}=I_{n}^{+}$,

$$
\left(H\left(I_{n}\right)-E\right) \psi(j)=\varepsilon^{2}(\Gamma \psi)(j),
$$

where $\Gamma=\partial I_{n}$. Therefore in $I_{n}^{+}$,

$$
\psi=a \psi_{n}^{+}+\varepsilon^{2} G_{n}^{\perp}(E) \Gamma \psi=a \psi_{n}^{+}+\mathcal{O}\left(\delta_{n}^{3}\right) .
$$

The last term above is obtained using $\frac{1}{2}\|\Gamma \psi\| \cong\left|\psi\left(c_{n}^{+} \pm l_{n} / 2\right)\right| \leq \exp -\gamma l_{n} / 2$ and $\left|G_{n}^{\perp}\left(E_{n}\right)\right| \leq 2 \delta_{n-1}^{-1}$ from Proposition 4.2. Similar estimates hold for $I_{n}^{-}$. These estimates together with the orthonormality of $\psi_{n+1}$ and $\tilde{\psi}_{n+1}$ yield (4.6). Now since any eigenfunction corresponding to $E$ in the interval $\left|E-E_{*}\right| \leq \delta_{n-1} / 3$ has the form (4.6), there can only be two such eigenvalues, $E_{n+1}, \tilde{E}_{n+1}$. The existence of these eigenvalues follows by using $\psi_{n}^{ \pm}$as trial wavefunctions,

$$
\left\|\left(H-E_{*}\right) \psi_{n}^{ \pm}\right\| \leq\left\|\Gamma \psi_{n}^{ \pm}\right\|+\left|E_{n}^{ \pm}-E_{*}\right| \leq \delta_{n}^{3}+\mathcal{O}\left(\delta_{n}^{1 / 2}\right) \ll \delta_{n-1} .
$$

See Remark c) following Theorem 2.1. 
The bound on $G_{n+1}^{\perp \perp}\left(E_{*}\right) \cong G_{n+1}^{\perp \perp}\left(E_{n+1}\right)$ follows from the fact that $E_{n+1}$ and $\tilde{E}_{n+1}$ are the only two eigenvalues in the interval $\left|E-E_{*}\right| \leq \delta_{n-1} / 3$.

Corollary 4.4. There is a unique eigenvalue $E_{n+1}$ for $H\left(I_{n+1}\right), I_{n+1} \in S_{n+1}$ such that $\left|E_{n+1}-E_{*}\right| \leq \delta_{n+1}^{1 / 3}$. Hence $E_{n+1}$ is well defined.

Proof. If $s_{n} \geq 4 l_{n}^{2}$ the result follows from Proposition 4.2. If $s_{n} \leq 4 l_{n}^{2}$ then Proposition 4.3 shows that $\psi_{n+1}$ and $\tilde{\psi}_{n+1}$ are supported in $I_{n}^{+} \cup I_{n}^{-}$which has width $l_{n+1}^{1 / 2} \cong l_{n}^{2}$. Therefore by Lemma 4.1 ,

$$
\left|E_{n+1}-\tilde{E}_{n+1}\right| \geq \exp -\beta l_{n+1}^{1 / 2} \geq \delta_{n+1}^{1 / 3} \equiv \exp \left(\frac{-\beta l_{n+1}^{2 / 3}}{3}\right) .
$$

Lemma 4.5. If $s_{n} \geq 4 l_{n}^{2}$ then for $\left|\theta-\theta_{*}\right| \leq \delta_{n} / 3$

$$
\left|\frac{d^{r}}{d \theta^{r}}\left(E_{n+1}(\theta)-E_{n}(\theta)\right)\right| \leq \delta_{n}^{3-\frac{r}{2}} \quad r=0,1,2 .
$$

Proof. By Proposition 4.2, $\left|\psi_{n+1}(j)\right| \leq \exp -\gamma l_{n} / 3$ for $j$ outside $I_{n}$. For $j$ inside $I_{n}$ we have as in the derivation of (4.7),

$$
\psi_{n+1}=a \psi_{n}+\varepsilon^{2} G_{n}^{\perp}\left(E_{n+1}\right) \Gamma_{n} \psi_{n+1},
$$

where $\Gamma_{n}=\partial I_{n}$. By Corollary $4.4\left\|G_{n}^{\perp}\left(E_{n+1}\right)\right\| \leq \delta_{n}^{-1}$ since $\left|E_{n+1}-E_{*}\right| \leq \delta_{n+1}$. Thus the last term of (4.9) is bounded by $\delta_{n}^{-1} e^{-\gamma l_{n} / 2} \leq \delta_{n}^{4}$ hence

$$
\left\|\psi_{n+1}-\psi_{n}\right\| \leq \delta_{n}^{4} \text {. }
$$

This bound and (2.10) yield (4.8) for $r=0,1$.

For $r=2$ we shall use (2.11),

$$
E^{\prime \prime}=\left\langle\psi, v^{\prime \prime} \psi\right\rangle+2\left\langle\psi, v^{\prime} G^{\perp}(E) v^{\prime} \psi\right\rangle
$$

We must estimate

$$
\begin{aligned}
G_{n+1}^{\perp}\left(E_{n+1}\right)-G_{n}^{\perp}\left(E_{n}\right)= & G_{n+1}^{\perp}\left(E_{n+1}\right)\left[\Gamma_{n}+\left(E_{n+1}-E_{n}\right)\right] G_{n}^{\perp}\left(E_{n}\right) \\
& -G_{n+1}^{\perp}\left(E_{n+1}\right) P_{n+1}^{\perp} P_{n}+G_{n}^{\perp}\left(E_{n}\right) P_{n}^{\perp} P_{n+1},
\end{aligned}
$$

restricted to $I_{n}$. This equation follows from the resolvent identity. We have used the orthogonal projections $P_{n}$ and $P_{n+1}$ onto $\psi_{n}$ and $\psi_{n+1}$ respectively and the relation $P_{n}+P_{n}^{\perp}=I=P_{n+1}+P_{n+1}^{\perp}$. The last two terms of (4.11) are bounded using (4.10).

$$
\left\|P_{n+1}^{\perp} P_{n}\right\|=\left\|P_{n}-P_{n+1} P_{n}\right\|=\left\|\psi_{n}-P_{n+1} \psi_{n}\right\|=\mathcal{O}\left(\delta_{n}^{4}\right),
$$

and

$$
\left\|G_{n}^{\perp}\left(E_{n}\right)\right\|=\mathcal{O}\left(\delta_{n}^{-1}\right)=\left\|G_{n+1}^{\perp}\left(E_{n+1}\right)\right\|,
$$

which follows from Corollary 4.4 and Proposition 4.2. The second term on the right side of (4.11) is bounded similarly since $\left|E_{n}-E_{n+1}\right| \leq \mathcal{O}\left(\delta_{n}^{4}\right)$, by (4.10). Therefore the case $r=2$ follows if we prove

$$
\left\|\Gamma_{n} G_{n}^{\perp}\left(E_{n}\right) v^{\prime} \psi_{n}\right\| \leq \delta_{n}^{3} .
$$


Let $\chi_{n}$ be the characteristic function of the interval

$$
\left\{j:\left|c_{n}-j\right| \leq l_{n} / 4\right\} \subset I_{n} .
$$

By the decay of $\psi$ given in Propositions 4.2 and 4.3 we have

$$
\left\|\left(1-\chi_{n}\right) v^{\prime} \psi_{n}\right\| \leq \mathcal{O}\left(\delta_{n}^{4}\right) .
$$

To prove $\left\|\Gamma_{n} G_{n}^{\perp} \chi_{n}\right\|$ is small let $A=I_{n} \backslash I_{n-1}$ or $I_{n} \backslash\left(I_{n-1}^{+} \cup I_{n-1}^{-}\right)$and $\Gamma=\partial I_{n-1}$ or $\partial\left(I_{n-1}^{+} \cup I_{n-1}^{-}\right)$depending on whether $I_{n}$ contains one or two singular intervals. Then

$$
\Gamma_{n} G_{n}^{\perp} \chi_{n}=\Gamma_{n} G_{A} \chi_{n}+\Gamma_{n} G_{A} \Gamma G_{n}^{\perp} \chi_{n}-\Gamma_{n} G_{A} P_{n} \chi_{n} .
$$

Since $A$ is $n-1$ regular $G_{A}(E)$ decays exponentially fast for $\left|E-E_{*}\right| \leq \delta_{n-1} / 3$. Hence $\Gamma_{n} G_{A} \chi_{n}$ and $\Gamma_{n} G_{A} \Gamma$ are exponentially small and $\left\|G_{A}\left(E_{n}\right)\right\| \leq \mathcal{O}\left(\delta_{n-1}^{-1}\right)$. The first two terms on the right side of (4.12) are now clearly less than $\mathcal{O}\left(\delta^{3}\right)$. To estimate the final term we use $\left\|\Gamma_{n} G_{n} \chi_{n}\right\| \leq \delta_{n}^{3}$ and $\left\|G_{A}\left(E_{n}\right)\right\| \cdot\left\|\left(1-\chi_{n}\right) \psi_{n}\right\|$ $\leq \delta_{n}^{3}$.

In the continuum the results of this section hold provided $\left|\theta-\theta_{*}\right| \leq \delta_{n} / 4$ is replaced by $\left|\theta-\theta_{*}\right| \leq \delta_{n} / 16 \pi$. See Theorem (2.3) and (2.13). The proofs are almost exactly the same if we use (2.9), (3.3) and Theorem 2.3. For the case $n=0$ we use the results of Appendix C. These same remarks apply to our final section.

\section{Proof of Theorem 2.1}

In this section we shall assume condition $\mathscr{C}_{n}$ holds [see (2.6)] and then establish $\mathscr{C}_{n+1}$, thereby proving Theorem 2.1. To do this we shall express $E_{n+1}(\theta)$ in terms of $E_{n}(\theta)$. The resulting estimates on $\frac{d}{d \theta} E_{n+1}(\theta)$ and $\frac{d^{2}}{d \theta^{2}} E_{n+1}(\theta)$ enable us to justify (1.9) and hence $\mathscr{C}_{n+1}$.

First let us consider the case where $s_{n} \geq 4 l_{n}^{2}$. Recall that Lemma 4.5 implies that for $r=0,1,2$,

$$
\left|\frac{d^{r}}{d \theta^{r}}\left(E_{n+1}(\theta)-E_{n}(\theta)\right)\right| \leq \delta_{n}^{3-\frac{r}{2}} \text { for }|\theta-\theta *| \leq \delta_{n} / 3 .
$$

This is the key estimate for this case.

If $s_{n} \leq 4 l_{n}^{2}$ we shall need a few lemmas. Let $I_{n}^{+}, I_{n}^{-} \in \bar{S}_{n}$ denote the two subintervals of $I_{n+1} \in S_{n+1}$ which are centered at $c_{n}^{+}$, $c_{n}^{-}$, respectively. When $\theta=\theta_{s} \equiv-c_{n+1} \alpha=-\left(c_{n}^{+}+c_{n}^{-}\right) \alpha / 2, H\left(I_{n}^{ \pm}\right)$are mirror images of each other. In fact

$$
v\left(j+c_{n}^{+}, \theta_{s}+\psi\right)=v\left(-j+c_{n}^{-}, \theta_{s}-\psi\right),
$$

and so

$$
\frac{d}{d \theta}\left(E_{n}^{+}+E_{n}^{-}\right)\left(\theta_{s}\right)=0 .
$$

Note that by $\mathscr{C}_{n}$ and (2.2)

$$
2\left|\theta_{*}-\theta_{s}\right| \equiv\left|\left(c_{n}^{+}+c_{n}^{-}\right) \alpha+2 \theta_{*}\right| \leq 12 \delta_{n}^{1 / 2} .
$$


Also by the remark following Theorem 2.1, since $s_{n} \leq 4 l_{n}^{2}$ we have $s_{n-1} \geq 4 l_{n-1}^{2}$ so that $I_{n}^{ \pm}$contains a single interval $I_{n-1}^{ \pm}$of $S_{n-1}$. Now we measure the deviation of (5.2) away from $\theta=\theta_{s}$.

Lemma 5.1. For $\left|\theta-\theta_{*}\right| \leq \delta_{n} / 3$ and $n \geq 1$,

$$
\left|\frac{d}{d \theta}\left(E_{n}^{+}+E_{n}^{-}\right)\right| \leq \mathcal{O}\left(\delta_{n}^{1 / 2} / \delta_{n-1}\right) .
$$

Proof. At $\theta=\theta_{s}$ the left side of (5.3) is 0 . Since $\left|\theta_{s}-\theta_{*}\right| \leq 6 \delta_{n}^{1 / 2}$ we have $\left|\theta-\theta_{s}\right| \leq 7 \delta_{n}^{1 / 2}$. By Proposition 4.2 and (2.11) for $\left|\theta-\theta_{*}\right| \leq \delta_{n-1} / 3$,

$$
\left|\frac{d^{2}}{d \theta^{2}} E_{n}^{ \pm}(\theta)\right|=\left|\left\langle\psi_{n}^{ \pm}, v^{\prime \prime} \psi_{n}^{ \pm}\right\rangle \sim 2\left\langle\psi_{n}^{ \pm}, v^{\prime} G_{n}^{\perp}\left(E_{n}^{ \pm}\right) v^{\prime} \psi_{n}^{ \pm}\right\rangle\right| \leq 2 \pi+6 \delta_{n-1}^{-1} .
$$

The lemma now follows by expressing the left side of (5.3) as an integral of the second derivative.

The following estimates, $\mathscr{D}_{n}$, will be established by induction on $n$ :

$\mathscr{D}_{n}:$ For $0 \leq m \leq n$ and $\left|\theta-\theta_{*}\right| \leq \delta_{m} / 4$,

$$
\left|\frac{d}{d \theta} E_{m}^{i}(\theta)\right| \geq \min \left[\delta_{m-1}^{2}, \frac{1}{2}\left|\theta+c_{m}^{i} \alpha\right|_{1}\right] .
$$

Recall that $\delta_{0}=2 \varepsilon$ and $\delta_{-1}=\varepsilon^{1 / 4}$.

Note that $d E_{m}^{i}(\theta) / d \theta$ vanishes at $\theta=-c_{m}^{i} \alpha$ by using the symmetry (1.8). $\mathscr{D}_{n}$ says that $E_{m}^{\prime}$ is small only near a symmetry point.

Lemma 5.2. If $s_{m} \leq 4 l_{m}^{2}$ and $\mathscr{D}_{m}$ holds then, for all $\theta$ such that $\left|\theta-\theta_{*}\right| \leq \delta_{m} / 4$,

$$
\left|\frac{d}{d \theta} E_{m}^{ \pm}(\theta)\right| \geq \delta_{m-1}^{2} \text {. }
$$

Proof. If (5.5) fails then $\mathscr{D}_{m}$ implies that for some $\theta$ in the interval $\left|\theta-\theta_{*}\right| \leq \delta_{m} / 4$ and some choice of + or - ,

$$
2 \delta_{m-1}^{2} \geq\left|\theta+c_{m}^{ \pm} \alpha\right|_{1}
$$

On the other hand, by (2.2)

$$
\left|\left(c_{m}^{+}+c_{m}^{-}\right) \alpha+2 \theta *\right|_{1} \leq 12 \delta_{m}^{1 / 2} .
$$

The above two estimates imply that $\left|\left(c_{m}^{+}-c_{m}^{-}\right) \alpha\right|_{1} \leq 3 \delta_{m-1}^{2}$ which violates the Diophantine condition (1.4), because $\left|c_{m}^{+}-c_{m}^{-}\right| \leq 4 l_{m}^{2}$.

Let $E^{\prime}(\theta), E^{\prime \prime}(\theta)$ denote the first and second derivatives of $E$ with respect to $\theta$. Recall that $\tilde{E}_{n+1}$ is the eigenvalue closest to $E_{n+1}$.

Lemma 5.3. Suppose $s_{n} \leq 4 l_{n}^{2}$ and $\mathscr{D}_{n}$ holds. If $\left|E_{n+1}^{\prime}(\theta)\right| \leq \delta_{n}$ for some $\left|\theta-\theta_{*}\right| \leq$ $\delta_{n} / 4$, then

and

$$
E_{n+1}^{\prime \prime}(\theta)=\frac{\left[d E_{n}^{+}(\theta) / d \theta\right]^{2}}{E_{n+1}(\theta)-\tilde{E}_{n+1}(\theta)}\left(1+\mathcal{O}\left(\delta_{n}\right)\right),
$$

$$
\left|E_{n+1}^{\prime \prime}(\theta)\right| \geq \frac{1}{3} \delta_{n}^{-2} \delta_{n-1}^{4} \geq 1
$$


and

$$
\left|\theta+c_{n+1} \alpha\right|_{1} \leq \text { const } \delta_{n}^{3 / 2}
$$

The same results hold with $E_{n+1}$ and $\tilde{E}_{n+1}$ interchanged.

Proof. We shall use expressions (2.11) and (2.12) to estimate $E_{n+1}^{\prime \prime}$. First note that by Proposition 4.3 and Lemma 5.1,

$$
\begin{aligned}
E_{n+1}^{\prime} & =\left\langle\psi_{n+1}, v^{\prime} \psi_{n+1}\right\rangle=A^{2}\left\langle\psi_{n}^{+}, v^{\prime} \psi_{n}^{+}\right\rangle+B^{2}\left\langle\psi_{n}^{-}, v^{\prime} \psi_{n}^{-}\right\rangle+\mathcal{O}\left(\delta_{n}^{2}\right) \\
& =A^{2} \frac{d}{d \theta} E_{n}^{+}(\theta)+B^{2} \frac{d}{d \theta} E_{n}^{-}(\theta)+\mathcal{O}\left(\delta_{n}^{2}\right) \\
& =\left(A^{2}-B^{2}\right) \frac{d}{d \theta} E_{n}^{+}(\theta)+\mathcal{O}\left(\delta_{n}^{1 / 2} / \delta_{n-1}\right) .
\end{aligned}
$$

Now if $\left|E_{n+1}^{\prime}(\theta)\right| \leq \delta_{n}$, then Lemma 5.2 and the above equation show that $A^{2} \cong B^{2} \cong 1 / 2$. Similarly we have

$$
\left|\left\langle\psi_{n+1}, v^{\prime} \tilde{\psi}_{n+1}\right\rangle\right|^{2} \cong\left|d E_{n}^{+} / d \theta\right|^{2} 4 A^{2} B^{2} \geq \frac{1}{2} \delta_{n-1}^{4}
$$

Next we claim that $\left|E_{n}^{ \pm}(\theta)-E_{n+1}(\theta)\right| \leq \mathcal{O}\left(\delta_{n}^{3}\right)$ whenever $|A| \cong|B|$. This result follows by taking the scalar product of $\psi_{n}^{ \pm}$with both sides of the identity

$$
\left(H\left(I_{n}^{ \pm}\right)-E_{n+1}\right) \psi_{n+1}=\varepsilon^{2} \Gamma_{n}^{ \pm} \psi_{n+1}=\mathcal{O}\left(\delta_{n}^{3}\right)
$$

hence

$$
\left\langle\psi_{n}^{ \pm}, \psi_{n+1}\right\rangle\left(E_{n}^{ \pm}-E_{n+1}\right)=A^{2}\left(E_{n}^{ \pm}-E_{n+1}\right)=\mathcal{O}\left(\delta_{n}^{3}\right)
$$

The last estimate follows from the decay of $\psi_{n+1}$ given by Proposition 4.3. A similar argument using $\tilde{\psi}_{n+1}, \tilde{E}_{n+1}$ shows that $\left|E_{n}^{ \pm}-\tilde{E}_{n+1}\right| \leq \mathcal{O}\left(\delta_{n}^{3}\right) \leq \delta_{n}^{2}$. Hence

$$
\left|E_{n+1}(\theta)-\tilde{E}_{n+1}(\theta)\right| \leq \mathcal{O}\left(\delta_{n}^{3}\right) \leq \delta_{n}^{2} .
$$

To obtain (5.8) note that since $\left|E_{n}^{+}(\theta)-E_{n}^{-}(\theta)\right| \leq \mathcal{O}\left(\delta_{n}^{3}\right), \mathscr{C}_{n}$ implies that

$$
2\left|c_{n+1} \alpha+\theta\right|_{1}=\left|\left(c_{n}^{+}+c_{n}^{-}\right) \alpha+2 \theta\right|_{1}=\mathcal{O}\left(\delta_{n}^{3 / 2}\right) \text {. }
$$

The proof of (5.6) and (5.7) is now complete using (5.9) and (5.10) to bound the first term on the right-hand side of (2.12). The last term of (2.12) is bounded using the bound $\left\|G_{n}^{\perp \perp}\right\|=\mathcal{O}\left(\delta_{n-1}^{-1}\right)$ of Proposition 4.3.

Remarks. If $E_{n+1}\left(\theta_{*}\right) \geq \tilde{E}_{n+1}\left(\theta_{*}\right)$, then this relation holds for all $\theta$, since no level crossing can occur. Hence (5.6) shows that $E_{n+1}^{\prime}(\theta)$ is increasing and $\tilde{E}_{n+1}^{\prime}(\theta)$ is decreasing whenever $\left|E_{n+1}^{\prime}(\theta)\right| \leq \delta_{n}$ and $\left|\theta-\theta_{*}\right| \leq \delta_{n} / 4$. Also both $E_{n+1}$ and $\tilde{E}_{n+1}$ are symmetric about $\theta_{s}$ (see Fig. 2). It is important to note that $E_{n+1}^{\prime}$ cannot re-enter the band $\left|E_{n+1}^{\prime}\right| \leq \delta_{n}$, since it is increasing there. Hence the set of $\theta$ 's where $\left|\theta-\theta_{*}\right| \leq \delta_{n} / 4$ and $\left|E_{n+1}^{\prime}\right| \leq \delta_{n}$ is an interval. If $E_{n+1} \leq \tilde{E}_{n+1}$ then the same arguments apply with $E, \tilde{E}$ interchanged. 


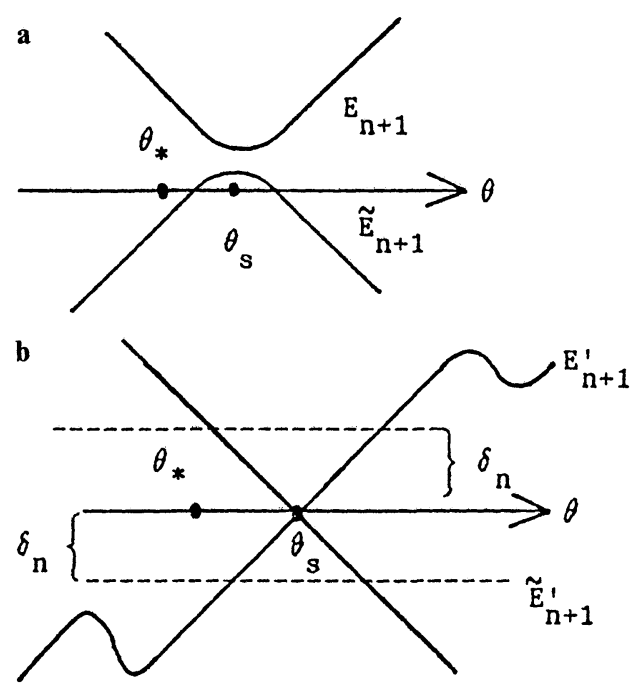

Fig. 2a and b. Graphs of $E_{n+1}, \tilde{E}_{n+1}$ and of $E_{n+1}^{\prime}, \tilde{E}_{n+1}^{\prime}$ restricted to $\left|\theta-\theta_{*}\right| \leq \delta_{n} / 4$, assuming $s_{n} \leq 4 l_{n}^{2}$. Note that $\theta_{s}=-c_{n+1} \alpha$

Lemma 5.4. Suppose $s_{n} \geq 4 l_{n}^{2}$ and that $\mathscr{D}_{n}$ holds. If $\left|E_{n+1}^{\prime}(\theta)\right| \leq \delta_{n}$ for some $\theta$, $\left|\theta-\theta_{*}\right| \leq \delta_{n} / 4$, then $\left|E_{n+1}^{\prime \prime}(\theta)\right| \geq 2 / 3$ and $E_{n+1}^{\prime \prime}(\theta)$ has the same sign for all such $\theta$.

Proof. Let $p$ be the largest value of $m \leq n$ such that $s_{m} \leq 4 l_{m}^{2}$. Then by (5.1), $\left|E_{n+1}^{\prime}(\theta)\right| \cong\left|E_{p+1}^{\prime}(\theta)\right| \leq \delta_{p+1}$, hence (5.7) implies that $\left|E_{p+1}^{\prime \prime}(\theta)\right|$ is large. Applying (5.1) again, we conclude $\left|E_{n+1}^{\prime \prime}(\theta)\right|$ is large. If $s_{m} \geq 4 l_{m}^{2}$ for all $m$ we use the fact that $\left|E_{0}^{\prime \prime}(\theta)\right| \geq 5 / 6$ and apply (5.1).

We now establish $\mathscr{D}_{n}$ [see (5.4)] by induction.

Lemma 5.5. $\mathscr{D}_{n+1}$ holds.

Proof. $\mathscr{D}_{0}$ clearly holds since the critical points of the cosine are nondegenerate. Now suppose $\mathscr{D}_{m}$ holds and that $\left|E_{m+1}^{\prime}(\theta)\right| \leq \delta_{m}^{2}$ for some $\theta$ such that $\left|\theta-\theta_{*}\right| \leq$ $\delta_{m+1} / 4$. Since $E_{m+1}^{\prime}\left(\theta_{s}\right)=0$, where $\theta_{s}=-c_{m+1} \alpha$, Lemmas 5.3 and 5.4 imply that

$$
\left|E_{m+1}^{\prime}(\theta)\right|=\left|E_{m+1}^{\prime \prime}(\xi)\right|\left|\theta-\theta_{s}\right| \geq \frac{1}{2}\left|\theta-\theta_{s}\right|,
$$

where $\xi$ is some point between $\theta_{s}$ and $\theta$. This establishes $\mathscr{D}_{m+1}$. In order to apply Lemma 5.3 and 5.4 we must check that $\left|\theta_{s}-\theta_{*}\right| \leq \delta_{m} / 4$. When $s_{m} \leq 4 l_{m}^{2}$ this follows easily from (5.8). If $s_{m} \geq 4 l_{m}^{2}$ then (5.1) implies that $\left|E_{m+1}^{\prime}(\theta)\right| \cong\left|E_{m}^{\prime}(\theta)\right| \leq$ $\delta_{m}^{2}$. By induction $\mathscr{D}_{m}$ implies that $\left|\theta-\theta_{s}\right| \leq \delta_{m}^{2}$ and since $\left|\theta-\theta_{*}\right| \leq \delta_{m+1}$, $\left|\theta_{s}-\theta_{*}\right| \leq \delta_{m} / 4$ follows. Finally note that by the remarks following Lemma 5.3 $\xi$ clearly belongs to the subinterval of $\left|\phi-\theta_{*}\right| \leq \delta_{m} / 4$, where $\left|E_{m+1}^{\prime}(\phi)\right| \leq \delta_{m}$ hence the lemmas can be applied as above.

The next lemma gives a weak form of $\mathscr{C}_{n+1}$ and uses only the results of Sect. 4 and $\mathscr{C}_{n}$. 
Lemma 5.6. If $c_{n+1}^{i}$ denotes the centers of $S_{n+1}$, then

$$
m\left(c_{n+1}^{i}, c_{n+1}^{j}\right)^{2} \leq \text { const } \delta_{n}^{3} .
$$

Proof. Let $I_{n+1}^{k}, k=i, j$ belong to $S_{n+1}$ and centered at $c_{n+1}^{k}$. Let $E_{n+1}^{k}$ be the eigenvalue of $H\left(I_{n+1}^{k}\right)$ closest to $E_{*}$. First suppose that $s_{n} \geq 4 l_{n}^{2}$. By (5.1)

$$
\left|E_{n}^{i}-E_{n}^{j}\right| \leq\left|E_{n}^{i}-E_{n+1}^{i}\right|+\left|E_{n+1}^{i}-E_{n+1}^{j}\right|+\left|E_{n+1}^{j}-E_{n}^{j}\right| \leq 2 \delta_{n}^{3}+2 \delta_{n+1},
$$

hence $\mathscr{C}_{n}$ implies that $m^{2}\left(c_{n}^{i}, c_{n}^{j}\right) \leq 3 \delta_{n}^{3}$. Since $c_{n+1}^{k}=c_{n}^{k}$ the lemma is proved for this case. Now suppose $s_{n} \leq 4 l_{n}^{2}$. Then for each $k$,

$$
\left|E_{n}^{2 k}-E_{n+1}^{k}\right| \text { or }\left|E_{n}^{2 k+1}-E_{n+1}^{k}\right| \leq \mathcal{O}\left(\delta_{n}^{3}\right),
$$

where $I_{n}^{2 k}, I_{n}^{2 k+1} \subset I_{n+1}^{k}$ are the two singular intervals in $\bar{S}_{n}$. This is easily established using $\psi_{n+1} \uparrow I_{n}^{2 k}$ or $\psi_{n+1} \uparrow I_{n}^{2 k+1}$ as a trivial wave function together with the estimates of Proposition 4.3. See the argument leading to (5.10). Let us label the centers $c_{n}^{2 k+1}=c_{n}^{2 k}+s_{n}$. Then it follows from $\mathscr{C}_{n}$ that either $m\left(c_{n}^{2 i}, c_{n}^{2 j}\right)$ or $m\left(c_{n}^{2 i}, c_{2 n}^{2 j+1}\right)<$ const $\delta_{n}^{3 / 2}$. By (2.2) we know that for $k=i, j$,

$$
\left|\left(c_{n}^{2 k}+c_{n}^{2 k+1}\right) \alpha+2 \theta *\right|_{1}=\left|\left(2 c_{n}^{2 k}+s_{n}\right) \alpha+2 \theta *\right|_{1} \leq \mathcal{O}\left(\delta_{n}^{1 / 2}\right),
$$

which implies that $\left|\left(c_{n}^{2 j}-c_{n}^{2 i}\right) \alpha\right|_{1} \leq \mathcal{O}\left(\delta_{n}^{1 / 2}\right)$. If we use this estimate together with those on $m$ above we obtain

$$
\left|\left(c_{n}^{2 i}-c_{n}^{2 j}\right) \alpha\right|_{1} \leq C \delta_{n}^{3 / 2} \quad \text { or } \quad\left|\left(c_{n}^{2 i}+c_{n}^{2 j+1}\right) \alpha+2 \theta *\right|_{1} \leq C \delta_{n}^{3 / 2} .
$$

Note that for example the case

$$
\left|\left(c_{n}^{2 i}-c_{n}^{2 j+1}\right) \alpha\right| \leq C \delta_{n}^{3 / 2}
$$

is excluded because it and $\left|\left(c_{n}^{2 j}-c_{n}^{2 i}\right) \alpha\right|_{1} \leq \mathcal{O}\left(\delta_{n}^{1 / 2}\right)$ would imply $\left|s_{n} \alpha\right|_{1} \leq \mathcal{O}\left(\delta_{n}^{1 / 2}\right)$ which violates the Diophantine properties of $\alpha$. Since $c_{n+1}^{k}=c_{n}^{2 k}+\frac{1}{2} s_{n},(5.12)$ follows from (5.13).

The proof of the following elementary lemma will be left to the reader.

Lemma 5.7. Let $E(\theta)$ be an even $C^{2}$ function for $\theta \in[a, b]$ such that whenever $\left|E^{\prime}(\theta)\right| \leq \delta$, then $\left|E^{\prime \prime}(\theta)\right| \geq 2 / 3$ and $E^{\prime \prime}(\theta)$ has a single sign for all such $\theta$. Then

$$
\left|E(\theta)-E\left(\theta^{\prime}\right)\right| \geq \frac{1}{4} M^{2}\left(\theta, \theta^{\prime}\right) \equiv \frac{1}{4} \min \left(\left|\theta+\theta^{\prime}\right|,\left|\theta-\theta^{\prime}\right|\right)^{2},
$$

provided $M\left(\theta, \theta^{\prime}\right) \leq \delta / 4$.

We shall set $E_{n+1}\left(\theta-c_{n+1} \alpha\right)=E(\theta)$ and $\delta=\delta_{n}$. Note that $E(\theta)=E(-\theta)$ follows from (1.8). The hypothesis of Lemma 5.7 is met using Lemmas 5.3-5.6, where $[a, b]=\left[\theta_{*}-\delta_{n} / 4, \theta_{*}+\delta_{n} / 4\right]$.

Proof of Theorem 2.1. We claim that as in (1.7),

$$
E_{n+1}^{j}\left(\theta_{*}+m\right)=E_{n+1}^{i}\left(\theta_{*}\right), \quad \tilde{E}_{n+1}^{j}\left(\theta_{*}+m\right)=\tilde{E}_{n+1}^{i}\left(\theta_{*}\right)
$$


where $|m|=m\left(c_{n+1}^{i}, c_{n+1}^{j}\right)$. By Lemma $5.6|m| \leq c \delta_{n}^{3 / 2}$. Clearly $E_{n+1}^{j}\left(\theta_{*}+m\right)$ is an eigenvalue of $H\left(I_{n+1}^{i}\right)$, by (1.7). To identify it with $E_{n+1}^{i}\left(\theta_{*}\right)$, note that using (5.12) and the bound $|d E(\theta) / d \theta| \leq 1$,

$$
\begin{aligned}
\left|E_{n+1}^{j}\left(\theta_{*}+m\right)-E_{*}\right| & \leq\left|E_{n+1}^{j}\left(\theta_{*}+m\right)-E_{n+1}^{j}\left(\theta_{*}\right)\right|+\left|E_{n+1}^{j}\left(\theta_{*}\right)-E_{*}\right| \\
& \leq C \delta_{n}^{3 / 2}+\delta_{n}^{3} \leq \delta_{n} / 3 .
\end{aligned}
$$

By Propositions 4.2 and 4.3 we conclude that $E_{n+1}^{j}\left(\theta_{*}+m\right)$ equals either $E_{n+1}^{i}\left(\theta_{*}\right)$ or $\tilde{E}_{n+1}^{i}$. The geometric structure of $E$ and $\tilde{E}$ (see Fig. 2) now allows us to conclude that (5.14) holds, so that in particular $E_{n+1}^{j}\left(\theta_{*}+m\right) \neq \tilde{E}_{n+1}^{i}\left(\theta_{*}\right)$. Since $E\left(\theta+c_{n}^{j}\right) \equiv E_{n+1}^{j}(\theta)$, where $E$ satisfies the previous lemma,

$$
\begin{aligned}
2 \delta_{n+1} & \geq\left|E_{n+1}^{i}\left(\theta_{*}\right)-E_{n+1}^{j}\left(\theta_{*}\right)\right|=\left|E_{n+1}^{j}\left(\theta_{*}+m\right)-E_{n+1}^{j}\left(\theta_{*}\right)\right| \\
& =\left|E\left(\theta_{*}+c_{n+1}^{i} \alpha\right)-E\left(\theta_{*}+c_{n+1}^{j} \alpha\right)\right| \geq \frac{|m|^{2}}{4},
\end{aligned}
$$

where $m=\left(c_{n+1}^{i}-c_{n+1}^{j}\right) \alpha$ or $m=-\left[2 \theta_{*}+\left(c_{i}+c_{j}\right) \alpha\right]$. This completes our induction.

\section{Appendix A. Proof of Theorem 2.2}

The proof of Theorem 2.2 is by induction on $n$. For $n=0$, see Sect. 1 . Now let $\Lambda$ be $n$-regular. We shall first suppose that $\Lambda$ contains a single $n$-regular interval $I_{n}$ and possibly many other $m$-regular intervals for $m<n$. The interval $I_{n}$ contains either a single interval $I_{n-1} \in S_{n-1}$ or two intervals $I_{n-1}^{-}, I_{n-1}^{+}$in $\bar{S}_{n-1}$. There is an interval $\bar{I}_{n-1} \subset I_{n}$ which contains $I_{n-1}^{+} \cup I_{n-1}^{-}$or $I_{n-1}$ such that
a) $A \equiv \Lambda \backslash \bar{I}_{n-1}$ is $(n-1)$-regular,
b) $\operatorname{dist}(\partial A,\{x, y\}) \geq l_{n-1}$,
c) length $\bar{I}_{n-1} \leq 5 l_{n}^{1 / 2}$.

This set is easily constructed using Lemma 2.4. If we set $G=G_{\Lambda}$ and $\bar{G}=G_{\Lambda, \bar{I}_{n-1}}$ then by (2.8) with $\Gamma=\partial A$

$$
G(x, y)=\{\bar{G}+\varepsilon \bar{G} \Gamma G\}(x, y)=\left\{\bar{G}+\varepsilon^{2} \bar{G} \Gamma \bar{G}+\varepsilon^{4} \bar{G} \Gamma G \Gamma \bar{G}\right\}(x, y) .
$$

Both $x$ and $y$ cannot belong to $\bar{I}_{n-1}$ since that would violate $|x-y| \geq l_{n}^{5 / 6}$, by c). First consider the case where $x, y \in A$, then $\bar{G}$ can be replaced by $G_{A}$. By applying the induction hypothesis to $G_{A}$ and expressing (A.1) in terms of its matrix elements we have

$$
\begin{aligned}
|G(x, y)| & \leq\left[\exp -\gamma_{n-1}|x-y|\right]+3 \exp \left[-\gamma_{n-1}\left(|x-y|-5 l_{n}^{1 / 2}\right)\right] \cdot \exp \beta l_{n}^{2 / 3} \\
& \leq \exp -\gamma_{n}|x-y|
\end{aligned}
$$

where $\gamma_{n}=\gamma_{n-1}-2 l_{n}^{2 / 3} / l_{n}^{5 / 6}$. Since $\Sigma l_{n}^{-1 / 6}$ is summable, we get a uniform lower bound on $\gamma_{n} \geq \frac{1}{2} \gamma_{0}=\frac{1}{2}\left|l_{n} \varepsilon\right|$ for $\varepsilon$ small. In (A.2) we have used a), b), c) above together with the bound $\|\Gamma G \Gamma\| \leq 2 \delta_{n}^{-1}=2 \exp \beta l_{n}^{2 / 3}$. To obtain this bound we use the fact that $I_{n}$ is regular, hence $\left\|G_{n}\right\| \equiv\left\|G_{I_{n}}(E, \theta)\right\| \leq 2 \delta_{n}^{-1}$, and we express $G$ using an alternating resolvent series

$$
G=G_{n}+\varepsilon^{2} G_{n} \Gamma_{n} G=G_{n}+\varepsilon^{2} G_{n} \Gamma_{n} \bar{G}+\varepsilon^{4} G_{n} \Gamma_{n} \bar{G} \Gamma G_{n}+\ldots
$$


Since $\left\|\Gamma_{n} \bar{G} \Gamma\right\|=\left\|\Gamma_{n} G_{A} \Gamma\right\| \leq \exp \left(-\gamma_{n-1} l_{n} / 2\right)$ holds by the induction hypothesis, our desired bound on $\|\Gamma G \Gamma\|$ follows from that on $\left\|G_{n}\right\|$. When $x$ or $y \in \bar{I}_{n}$ we use the first equality of (A.2) and the rest of the proof goes as above.

Note that the bound $\left\|G_{n}\left(E_{*}, \theta_{*}\right)\right\| \leq \delta_{n}^{-1}$ can be extended by using the resolvent series $\left\|G_{n}(E, \theta)\right\| \leq 3 \delta_{n}^{-1}$ provided that $\left\|v(\theta)-v\left(\theta_{*}\right)\right\|+\left|E-E_{*}\right| \leq \frac{2 \delta_{n}}{3}$. Hence the estimates of this appendix apply to those $\theta$ and $E$ specified in Theorem 2.2.

The general case where $\Lambda$ contains many $n$-regular interval $I_{n}^{\prime}$ is treated as in $[10,12]$. We use the fact that the intervals $I_{n}$ are separated by a distance $3 l_{n}$ and then express $G_{\Lambda}$ as a sum of products of Green's functions $G_{\Lambda^{\prime}}$ where $\Lambda^{\prime}$ contains a single $n$ regular interval. This is called the block resolvent expansion in [12]. Condition $\mathscr{C}_{n-1}$ is only needed to ensure the separation of the intervals $I_{m}^{i}, m \leq n-1$.

For our analysis of $H_{c}$ we apply (2.8) instead of (2.7) to obtain the identities corresponding to (A.1) and (A.3). Bounds on the derivatives of the Green's function follow by expressing $G(E)=G^{0}(E)-K^{2} G^{0}(E) v G(E)$, where $G^{0}(E)$ is the Green's function with $v$ set equal to zero. Since we consider $E \cong-2 K^{2}+\sigma(K)$, $G^{0}$ and its derivatives decay like $\exp (-|K||x-y|)$.

\section{Appendix B. Eigenvalue Splitting}

Proof of Lemma 4.1. We first consider the finite difference case. Let $\tilde{\psi}_{1}(x)=-\psi(x)$ for $x \leq a$ and $\tilde{\psi}_{1}(x)=\psi_{1}(x)$ for $x>a$. Then

$$
\begin{aligned}
\left\langle\tilde{\psi}_{1}, H \psi_{2}\right\rangle & =E_{2}\left\langle\tilde{\psi}_{1}, \psi_{2}\right\rangle \\
& =E_{1}\left\langle\tilde{\psi}_{1}, \psi_{2}\right\rangle+2 \varepsilon^{2}\left[\psi_{2}(a) \psi_{1}(a+1)-\psi_{1}(a) \psi_{2}(a+1)\right] .
\end{aligned}
$$

Let us normalize $\psi_{1}$, and $\psi_{2}$ so that for $i=1,2, \psi_{i}(a)=\cos \left(\theta_{i}\right), \psi_{i}(a+1)=\sin \theta_{i}$. Since the transfer matrix has norm less than $\left[\varepsilon^{-2}+4\right]$ we have

$$
\left\|\psi_{i}\right\| \leq 2\left\|\chi \psi_{1}\right\| \leq 3\left|\varepsilon^{-2}+4\right| .
$$

Therefore for small $\varepsilon$, (B.1) and the above inequality imply that

$$
\left|\sin \left(\theta_{1}-\theta_{2}\right)\right| \leq\left|E_{1}-E_{2}\right|\left\|\left\langle\tilde{\psi}_{1}, \psi_{2}\right\rangle\right\| \leq \frac{1}{4}\left|E_{2}-E_{1}\right|\left\|\psi_{1}\right\|^{1 / 2}\left\|\psi_{2}\right\|^{1 / 2}\left[\frac{\varepsilon^{2}}{2}\right]^{-(l+1)} .
$$

If $\left|E_{1}-E_{2}\right|$ is very small, then $\psi_{1}$ and $\psi_{2}$ satisfy nearly the same initial data and nearly the same equation. Thus $\psi_{1}$ and $\psi_{2}$ are nearly equal on the support of $\chi$, which contradicts the orthogonality of $\psi_{1}$ and $\psi_{2}$. In fact it is straightforward to see that

$$
\begin{aligned}
\left\|\chi\left(\psi_{1}-\psi_{2}\right)\right\| & \leq\left[\left|E_{1}-E_{2}\right| l+\left|\sin \left(\theta_{1}-\theta_{2}\right)\right|\right]\left[\varepsilon^{-2}+4\right]^{l} \\
& \leq\left|E_{1}-E_{2}\right|\left\|\psi_{1}\right\|^{1 / 2}\left\|\psi_{2}\right\|^{1 / 2}\left[\frac{\varepsilon^{2}}{2}\right]^{-2(l+1)}
\end{aligned}
$$


On the other hand $\left\|\psi_{1}\right\|^{2}+\left\|\psi_{2}\right\|^{2} \leq 2\left\|\chi\left(\psi_{1}-\psi_{2}\right)\right\|^{2}$ because

$$
\begin{aligned}
\left\|\psi_{1}\right\|^{2}+\left\|\psi_{2}\right\|^{2} & =\left\|\psi_{1}-\psi_{2}\right\|^{2}=\left\|\chi\left(\psi_{1}-\psi_{2}\right)\right\|^{2}+\left\|(1-\chi)\left(\psi_{1}-\psi_{2}\right)\right\|^{2} \\
& \leq\left\|\chi\left(\psi_{1}-\psi_{2}\right)\right\|^{2}+\frac{1}{2}\left(\left\|\psi_{1}\right\|^{2}+\left\|\psi_{2}\right\|^{2}\right) .
\end{aligned}
$$

In the last inequality we used (4.1). The lemma now follows by combining the above inequality and (B.3).

In the continuum we use the identity

$$
\frac{d}{d x}\left[\psi_{1}^{\prime}(x) \psi_{2}(x)-\psi_{1}(x) \psi_{2}^{\prime}(x)\right]=\left(E_{2}-E_{1}\right) \psi_{1}(x) \psi_{2}(x)
$$

to estimate $\left|E_{2}-E_{1}\right|$. Integrating both sides of (B.4) from $a$ to the endpoint of $\Lambda$ we obtain the analogue of (B.1),

$$
\left|\psi_{1}^{\prime}(a) \psi_{2}(a)-\psi_{1}(a) \psi_{2}^{\prime}(a)\right| \leq\left|E_{2}-E_{1}\right|\left\|\psi_{1}\right\|\left\|\psi_{2}\right\|
$$

We normalize $\psi_{i}(a)=\cos \theta_{i}, \psi_{i}^{\prime}(a)=\sin \theta_{i}$. Since $\left|E_{*}-V\right|^{1 / 2} \leq 2 K$, solutions grow at most like $\exp 2 K|x|$ and we obtain the analogue of (B.3), as above.

Remarks. This theorem uses the Dirichlet boundary condition at the boundary of $A$ in the derivation of B.1 and B.5. The theorem gives eigenvalue splittings for any bounded potential on the line in terms of the growth of the respective eigenfunctions.

\section{Appendix C. Estimates on $E_{0}^{i}$ (Continuum)}

In this appendix we shall establish (1.6) for the case $n=0$, and obtain bounds on $E_{0}^{\prime \prime}(\theta)$, where $E_{0}$ is the lowest eigenstate of $H\left(I_{0}\right)$ and $I_{0} \in S_{0}$. Let $v(x)$ be given by $(1.2)$ and let $v(x(\theta), \theta)$ be the minimum of $v(x, \theta)$ restricted to $I_{0}$. By definition of $S_{0}$,

$$
(1-\cos 2 \pi x(\theta))+(1-\cos 2 \pi(x(\theta) \alpha+\theta)) \leq \frac{12}{K} .
$$

This implies $x(\theta)$ must be nearly integer valued. In fact $|x(\theta)|_{1} \leq K^{-1 / 2}$ and $|x(\theta) \alpha+\theta *|_{1} \leq K^{-1 / 2}$, hence $2\left|c_{0} \alpha+\theta *\right|_{1} \leq 2 K^{-1 / 2(1+\alpha)}$. This yields (1.6) for $n=0$ with $\delta_{0}=K^{-1}$ provided $|\alpha| \leq 1$. Note that this also implies that the centers of $S_{0}$ are separated by at least $C_{1} K^{1 / 4} / 4$.

It is well-known that $E_{0}(\theta)$ has a standard asymptotic expansion

$$
\begin{aligned}
E_{0} & =K^{2} v(x(\theta), \theta)+K\left(v^{\prime \prime} / 2\right)^{1 / 2}+\mathcal{O}(1) \\
& =K^{2} v(x(\theta), \theta)+K \pi\left(2+2 \alpha^{2}\right)^{1 / 2}+\mathcal{O}(1),
\end{aligned}
$$

where

$$
v^{\prime \prime}(x(\theta), \theta)=\frac{d^{2}}{d x^{2}} v(x(\theta), \theta) \cong 4 \pi^{2}\left(1+\alpha^{2}\right) .
$$

Note that the next eigenvalue of $H\left(I_{0}\right)$ is larger than

$$
-2 K^{2}+3 \pi\left(2+2 \alpha^{2}\right)^{1 / 2} \geq-2 K^{2}+10 K .
$$

This explains our constraint on $E_{*}$ in Theorem 2.3 and allows us to establish (5.13) for $E_{0}^{i}$. 
Next we consider derivatives of $E(\theta)$. It is easy to check that $d x(\theta) / d \theta \cong$ $-\alpha /\left(1+\alpha^{2}\right)$. If we formally differentiate both sides of (C.2) we see that

$$
\frac{d^{2}}{d \theta^{2}} E_{0}(\theta)=(2 \pi K)^{2} /\left(1+\alpha^{2}\right)+\mathcal{O}(K)
$$

which is the desired lower bound. This identity may be justified using (2.11) and the asymptotics for $\psi$. This bound is used to establish (5.4) for the $n=0$ case.

Acknowledgements. We wish to thank J.Moser and B.Simon for their advice and encouragement. T.S. would also like to thank S. Surace for numerous discussions. J.F. and T.S. would like to thank M. Berger, K. Gawedzki and D. Ruelle for their hospitality at I.H.E.S. Finally we wish to thank A. Klein and F. Martinelli for a critical reading of this paper.

\section{References}

1. Sinai, Ya.: J. Stat. Phys. 46, 861 (1987)

2. Aubrey, S.: Solid Sci. 8, 264 (1978)

3. Avron, J., Simon, B.: Duke Math. J. 50, 369 (1983)

4. Herman, M.: Comment. Math. Helv. 58, 453 (1983)

5. Delyon, F.: J. Phys. A 20, L 21 (1987)

6. Gordon, A.: Usp. Math. Nauk 31, 257 (1976)

7. Bellissard, J., Lima, R., Testard, D.: Commun. Math. Phys. 88, 207 (1983)

8. Dinaburg, E., Sinai, Ya.; Funct. Anal. App. 9, 279 (1975)

9. Surace, S.: N.Y.U. Thesis (1987) to appear TRANS, AMS

10. Fröhlich, J., Spencer, T.: Commun. Math. Phys. 88, 151 (1983)

11. Fröhlich, J., Martinelli, F., Scoppola, E., Spencer, T.: Commun. Math. Phys. 101, 21 (1985)

12. Spencer, T.: In: Critical phenomena, random fields, gauge theories. Osterwalder, K., Stora, R. (eds.). Amsterdam: North Holland 1986

13. Berezanskii, J.: Expansion in eigenfunctions of self adjoint operators. Transl. Math. Mono. 17 (1968)

14. Simon, B.: Schrödinger semigroups. Bull. AMS 1, 447 (1983)

15. Kirsch, W., Simon, B.: Commun. Math. Phys. 97, 453 (1985)

Communicated by A. Jaffe

Received July 13, 1988; in revised form May 21, 1990 
